\title{
Therapy of Pancreatic Neuroendocrine Tumors: Fine Needle Intervention including Ethanol and Radiofrequency Ablation
}

\author{
Sundeep Lakhtakia \\ Department of Gastroenterology, Asian Institute of Gastroenterology, Hyderabad, Telangana, India
}

Pancreatic neuroendocrine tumors (PNETs) are increasingly being detected, though usually as incidental findings. Majority of the PNETs are non-functional and surgical resection is the standard of care for most of them. However, in patients with small PNETs localized within the pancreas, who are unfit or unwilling for surgery, alternate methods of treatment are needed. Direct methods of ablation of PNETs, using either ethanol injection or radiofrequency ablation (RFA), are emerging as effective methods. The limited literature available as case reports or case series on endoscopic ultrasound (EUS)-guided local ablation using either ethanol or RFA has demonstrated safety and efficacy along with short- to medium-term sustained relief. Long-term benefits with these local ablative therapies are awaited. Comparative studies are needed to show which of these two competing technologies is superior. Finally, comparative trials of EUS-guided ablation with surgical resection in terms of efficacy and safety will ensure their place in the management algorithm. Clin Endosc 2017;50:546-551

Key Words: Pancreatic neuroendocrine tumor; Endoscopic ultrasound; Radiofrequency ablation; Tumor ablation; Ethanol

\section{INTRODUCTION}

Neuroendocrine tumors (NETs) are increasingly being detected mainly due to better cross-sectional imaging and the frequent use of endoscopy for surveillance for cancer. The most frequent sites of origin for gastrointestinal (GI) NETs are the pancreas and small intestine. ${ }^{1}$ The incidence of pancreatic NETs (PNETs) smaller than $2 \mathrm{~cm}$ has remarkably increased. ${ }^{2}$

PNETs show wide heterogeneity in their biological behavior. $^{3}$ Clinically, based on symptoms, PNETs can be functional (10\%-30\%) or non-functional (70\%-90\%). Functional PNETs secrete biologically active peptides, or hormones, which may affect quality of life.

Received: October 20, 2017 Revised: November 15, 2017

Accepted: November 16, 2017

Correspondence: Sundeep Lakhtakia

Department of Gastroenterology, Asian Institute of Gastroenterology, 6-3-661 Somajiguda, Hyderabad, Telangana 500082, India

Tel: +91-40-2337-8888, Fax: +91-40-2332-4255,

E-mail: drsundeeplakhtakia@gmail.com

(c) This is an Open Access article distributed under the terms of the Creative Commons Attribution Non-Commercial License (http://creativecommons.org/ licenses/by-nc/3.0) which permits unrestricted non-commercial use, distribution, and reproduction in any medium, provided the original work is properly cited.
Surgical removal is the strategy of choice for management of PNETs in young and healthy patients, and for lesions with the main pancreatic duct involvement and local invasion (e.g., dilation of the main pancreatic duct, bile duct, vascular and nodal involvement). On the contrary, conservative management may be considered for asymptomatic, non-functioning PNETs of $<2 \mathrm{~cm}$ size in patients affected by multiple endocrine neoplasia type 1 and for selected patients (significant comorbidity, advanced age) with the sporadic types of PNETs. ${ }^{3}$

Pancreatic surgery may be associated with significant adverse events. The maximal acceptable post-operative complication rate after surgery for PNETs is up to $50 \%$. The maximal acceptable mortality rate for pancreatico-duodenectomy is $5 \%$ and for distal pancreatectomy is $1 \% .{ }^{3}$ Minimally invasive pancreatic resection of localized PNETs of the body/tail, using either laparoscopic or robotic techniques, may be considered in specialized centers. ${ }^{3}$ Patients, who are unfit for or refuse surgery, need an alternative non-surgical method to ablate these potentially malignant lesions and alleviate symptoms associated with functional PNETs.

For patients who are not eligible for surgical resection, the choice of treatment depends on the stage of the disease, 


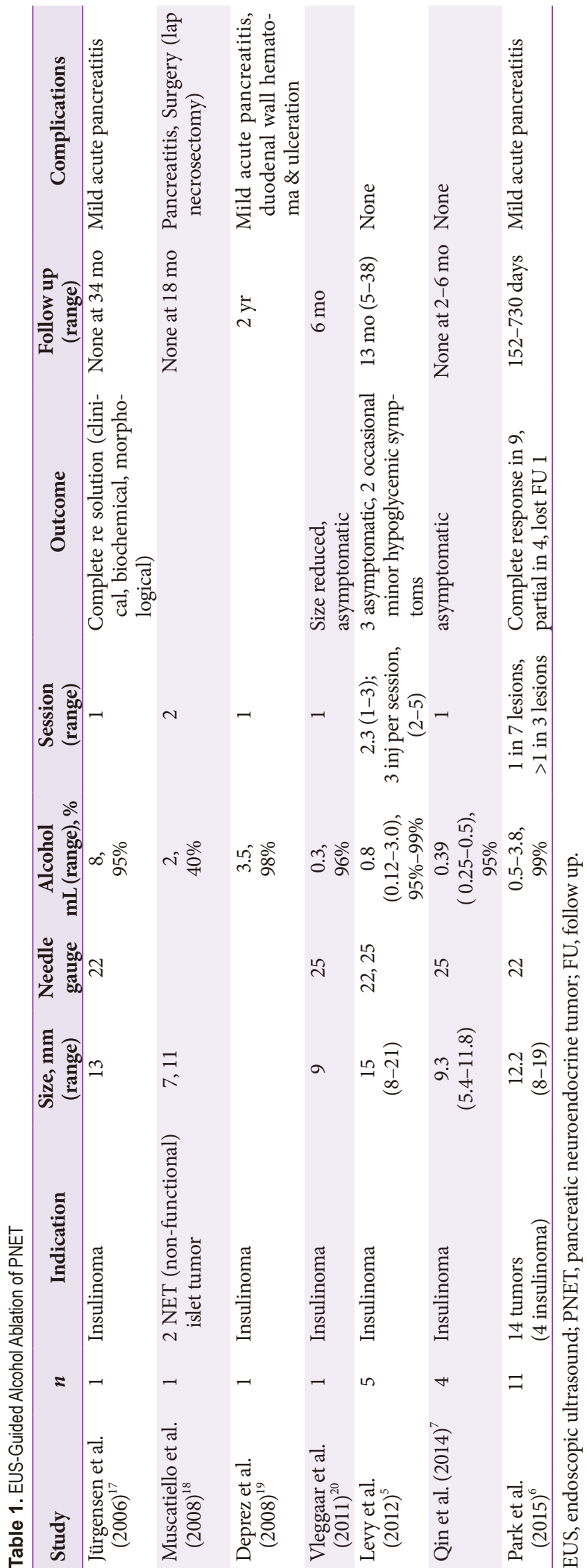

symptoms, and histological features of the tumor. Treatment options include somatostatin analogues and palliative therapies for metastatic liver disease (e.g., chemo-embolization, radio-embolization, arterial embolization and radiofrequency ablation [RFA]). ${ }^{4}$ Non-surgical treatment modalities for PNETs including direct tumor ablation using ethanol injection or RFA may be considered in patients unfit or not willing for surgery. ${ }^{5-10}$

Endoscopic ultrasound (EUS) is used to discover a functional NET (insulinoma) when conventional imaging fails to localize the lesion. Also, EUS helps in (1) guided fine needle aspiration (FNA) to confirm pathology, (2) tattoo with India ink for ease at intra-operative identification during surgery, and (3) for EUS guided tumor ablation. Fine needle intervention (FNI) techniques described for EUS-guided tumor ablation of PNETs include either ethanol injection or RFA.

\section{EUS-GUIDED ETHANOL ABLATION}

Ethanol ablation is widely accepted for the treatment of hepatocellular carcinoma (HCC) via the percutaneous route under trans-abdominal image guidance. ${ }^{11}$ EUS-guided ethanol injection in the liver was first reported for treatment of hepatic metastases from rectal adenocarcinoma. ${ }^{12}$ However, the technique has now been reported for management of GI stromal tumors ${ }^{13}$ and adrenal metastases from non-small-cell lung cancer. ${ }^{14}$

The feasibility of EUS-guided ethanol injection into normal pancreas was first reported in animal studies on pigs. ${ }^{15,16}$ Subsequently, the technique was used for ablation of pancreatic tumors in subjects with insulinoma or non-functional PNETs who were not eligible for surgical resection (Table 1). However, technical variables (optimal type of intervention needle and its caliber, volume of alcohol to be injected) and the technique itself to minimize the potential risk of pancreatitis remain non-uniform.

Jürgensen and colleagues were the first to publish their successful experience of EUS-guided ethanol injection into a patient with functional pancreatic NET (insulinoma). ${ }^{17}$ They used a total of $8 \mathrm{~mL}$ of $95 \%$ alcohol, which was injected into a 13-mm symptomatic insulinoma under EUS guidance. Complete resolution was reported based on clinical, morphologic, and biochemical data. ${ }^{16}$

Muscatiello et al. reported a patient with two PNETs, who underwent two sessions of ethanol ablation. ${ }^{18}$ Less than $2 \mathrm{~mL}$ of $40 \%$ ethanol was injected during each session. While there were no adverse events following the first session, the second session was associated with necrotizing pancreatitis requiring laparoscopic necrosectomy. ${ }^{18}$ At the two-month follow up, 
somatostatin receptor scintigraphy and biochemical measurement for peptides were normal.

Deprez et al. reported the case of an elderly woman with insulinoma in the pancreatic head who underwent EUS-guided ethanol ablation using $3.5 \mathrm{~mL}$ of $98 \%$ ethanol after placement of a pancreatic \& biliary stent. ${ }^{19}$ Post-procedure, there was a mild asymptomatic elevation of pancreatic enzymes with duodenal hematoma and ulceration that were treated conservatively. There was no recurrence of hypoglycemia for about two years after the ablation.

Vleggaar et al. reported the case of an elderly patient with a symptomatic insulinoma and co-morbidities who successfully underwent EUS-guided ethanol ablation. ${ }^{20}$ They injected 0.3 $\mathrm{mL}$ of $96 \%$ ethanol into the tumor located in the pancreatic body using a 25-gauge needle. There were no symptoms of hypoglycemia for up to six months after the ablation.

Levy and colleagues reported a series of 8 cases of symptomatic PNETs (insulinoma) who received an intra-lesional ethanol (99\%) injection either under EUS guidance (5) or under intra-operative ultrasound (IOUS)-guidance (3). ${ }^{5}$ All patients receiving the IOUS-guided injection understandably received a single injection each during surgery, whereas the patients receiving the EUS-guided FNI received $>1$ injections (mean 2.3, range 1-3). The volume of ethanol injected per session was smaller for the EUS guidance group (mean 0.8 $\mathrm{mL}$, range $0.12-3.0$ ) compared to the IOUS-guided group (1.2 $\mathrm{mL}$, range $0.8-1.5)$. No complications were reported in any of the 5 patients treated with the EUS-guided FNI. However, the IOUS-guided injection group had self-limiting complications such as minor bleeding in one and pseudocyst in another.

Park et al. published a report on the successful EUS-guided ethanol injections in 11 subjects who were poor surgical candidates having 14 PNETs of small size (non-functional 10, insulinoma 4). ${ }^{6}$ A single injection of ethanol (volume range, 0.5 to $3.8 \mathrm{~mL}$ ) resulted in a complete response at the 3-month radiologic imaging for 7 of the 13 tumors (response rate, $53.8 \%$ ), and the effect was maintained till up to the 1-year follow up. Multiple treatment sessions were required in 3 tumors which showed residual viability after one injection, thereby increasing the number of tumors with complete response to 8 out of 13 (response rate, 61.5\%). Mild pancreatitis occurred in 3 out of 11 patients.

\section{TECHNICAL ASPECTS OF EUS-GUIDED ETHANOL ABLATION}

EUS FNA needle (22 or $25 \mathrm{G}$ ) is advanced into the tumor (but not through it) and a very small volume of ethanol (about $0.1 \mathrm{~mL}$ ) is injected. The injections may be incrementally re- peated at the same site until a hyper-echoic blush is visible expanding within the tumor. Further injection of ethanol at any particular site is terminated when the hyper-echoic blush extends up to the margin of the tumor or if there is a likelihood of leakage beyond its border. For larger lesions, additional small injections are given in the same path as the needle is withdrawn for the remnant tumor. Before complete removal, the needle is held in place within the tumor for approximately 60 seconds to minimize tracking of ethanol into surrounding normal area. Based on the tumor size and pattern of spread after the initial injection, additional passes can be made avoiding the same needle tracts. The goal is to inject just enough ethanol to permeate the entire tumor, and terminate the injection as it appears to extravasate out. Needle with multiple side holes are to be avoided for ethanol injection.

In summary, literature has reports of patients who received one or more EUS-guided ethanol injections per session with small volumes of concentrated ethanol using a small caliber EUS FNA needle (22 or $25 \mathrm{G}$ ). The sessions may be repeated if imaging or symptoms indicate that the lesions persist. EUS-guided ethanol injection seems to have a role in patients with insulinomas or non-functional PNETs who are not eligible for surgical resection. Multicenter controlled studies with long term follow-up are needed to prove the efficacy of this procedure using defined inclusion criteria, methodology and end points.

\section{EUS-GUIDED RFA}

RFA uses high-frequency alternating current, which generates energy as heat that causes coagulative necrosis in the target tissue. RFA can be delivered by diverse approaches depending on the location and histology of the lesion: trans-cutaneous approach under radiological guidance for superficial HCC and focal liver metastases, intra-operative approach for deep seated lesions, endo-biliary route for inoperable bile duct malignancies or endoscopic transmural approach under EUS guidance for pancreatic or peri-luminal lesions. The major advantage with RFA is its minimally invasive nature and safety.

The requirement for a less invasive substitute to surgery and the success of RFA in hepatic tumors encouraged its use for pancreatic tumors. Pancreas, being a thermo-sensitive organ, thermal ablation of normal pancreas can lead to an inflammatory reaction causing edema, occasionally cystic transformation and later fibrosis. There is a growing interest and need for RFA in diverse focal lesions of the pancreas, including PNETs and unresectable pancreatic carcinomas where it is feasible. Earlier animal experiments with EUS 
RFA have demonstrated reasonable safety. ${ }^{21-23}$

Currently, there are two groups varieties of EUS RFA delivery devices. ${ }^{24}$

Through-the-needle device (e.g., Habib ${ }^{\mathrm{TM}}$ EUS-RFA catheter; EMcision Ltd., London, UK) is a very slender soft metallic wire, which is insulated except for the $1-2 \mathrm{~cm}$ long tip that delivers local energy (1 French caliber, $0.33 \mathrm{~mm}$, or 0.013 inch thick, $190 \mathrm{~cm}$ long). It is passed through the hollow of an FNA needle (22 or $19 \mathrm{G}$ ) and manipulated to project beyond the tip. Fluoroscopy assists in visualization of the projecting wire. The tip of this probe is floppy, and may take a curled shape.

Needle RFA device (e.g., EUSRA from STARmed, Korea or HybridTherm Probe [HTP], from ERBE) resembles the conventional EUS FNA needle. They have a thicker caliber (18/19 or $14 \mathrm{G}$, respectively) and an insulated covering except for the variable length of exposed active electrode at the tip. Both these devices have the facility for internal cooling to prevent charring of its metal surface.

Each device can be connected at the handle to their respective sophisticated generator which delivers accurate energy. The controlled heating of the target lesion can be visualized real-time with EUS as the appearance of echogenic bubbles around the needle tip. More than one zone in the lesion can be ablated depending on its size by either withdrawing and burning the more proximal lesion in the same trajectory of the needle path or in a different trajectory by either fanning or re-puncturing, the same lesion. ${ }^{8}$

Two of the three currently available EUS RFA devices have been clinically used for ablation of pancreatic NETs (Habib EUS RFA, EUSRA RF Electrode; STARmed, Goyang, Korea). The third, HTP (ERBE Elektromedizin GmbH, Tübingen, Germany); a bipolar probe with a longer $(25 \mathrm{~mm})$ active electrode has not been reported for generally small sized PNETs.

The overall clinical experience with EUS-guided RFA for PNETs is limited. In a recent review by Alvarez-Sánchez et al., a total of 42 patients from several case series were reported to have undergone EUS-guided RFA for a variety of pancreatic lesions. ${ }^{8-10,25-29}$ Out of these, 7 patients had PNETs (Table 2). Among patients with PNETs, three had symptomatic insulinoma. ${ }^{8}$

Armellini et al. first reported successful ablation in an elderly patient who rejected surgical resection, by EUS-guided RFA of a non-functional $2 \mathrm{~cm}$ PNET located in the pancreatic tail, using an $18 \mathrm{G}$ EUSRA (STARmed) in a single session. ${ }^{26}$ There were no procedure related complications. At the one-month follow-up, computed tomography (CT) scan and contrast-enhanced EUS confirmed complete resolution.

Rossi et al. performed ultrasound-guided RFA in 10 patients with histologically diagnosed PNETs (functional 3) who were not eligible for surgery. ${ }^{28}$ The mean size of the lesions was $1.6 \mathrm{~cm}$ (range $0.9-2.9 \mathrm{~cm}$ ), and they were located in the head (7) or body (3) of the pancreas. Only one subject underwent EUS-guided RFA, and the others received ultrasound guidance percutaneously (7) or intra-operatively (2). They defined 'complete ablation' as the absence of enhancing tissue at the tumor site on contrast-enhanced imaging studies and normalization of previously elevated serum hormone levels. Majority of the lesions were ablated following a single session (9), and only one patient required 2 sessions. The hormone-related symptoms regressed within a day of treatment. Complications included acute pancreatitis in 3 patients, of which 2 developed pancreatic fluid collections requiring endoscopic drainage. There were no recurrences at the 3-year follow up.

Pai et al. in their pilot study included 8 patients with diverse pancreatic lesions for EUS-guided RFA using a monopolar RF probe (1.2 mm Habib EUS-RFA catheter) placed through a 19 or $22 \mathrm{G}$ FNA needle. ${ }^{10}$ Two out of the 8 subjects had PNETs, both located in the head, with a mean size of $27.5 \mathrm{~mm}$. While one patient received 6 applications in one session, the other received 4 applications in two sessions. There was a reduction in the vascularity and appearance of central necrosis after

Table 2. EUS-Guided RFA for PNET

\begin{tabular}{|c|c|c|c|c|c|c|c|c|c|}
\hline Study & $n$ & Indication & $\begin{array}{l}\text { Size, } \mathrm{mm} \\
\text { (range) }\end{array}$ & RF device & $\begin{array}{l}\text { Thermo- } \\
\text { kinetics }\end{array}$ & $\begin{array}{c}\text { RF } \\
\text { session }\end{array}$ & $\begin{array}{l}\text { Outcome: } \\
\text { ablation }\end{array}$ & $\begin{array}{l}\text { Recur- } \\
\text { rence }\end{array}$ & $\begin{array}{l}\text { Compli- } \\
\text { cations }\end{array}$ \\
\hline $\begin{array}{l}\text { Armellini et al. } \\
(2015)^{26}\end{array}$ & 1 & PNET & 20 & $\begin{array}{c}18 \mathrm{G}, \\
\text { Starmed }\end{array}$ & - & 1 & Complete & - & None \\
\hline Rossi et al. $(2014)^{28}$ & 1 & PNET & 9 & $\begin{array}{l}\text { Habib EUS } \\
\text { RFA }\end{array}$ & $10-15 \mathrm{~W}$ & 1 & Complete & $\begin{array}{l}\text { None at } 34 \\
\text { mo }\end{array}$ & None \\
\hline Pai et al. $(2015)^{10}$ & 2 & PNET & $\begin{array}{c}27 \\
(15-40)\end{array}$ & $\begin{array}{l}\text { Habib EUS } \\
\text { RFA }\end{array}$ & $\begin{array}{c}20 \mathrm{~W} \\
90-120 \mathrm{secs}\end{array}$ & 1,2 & $\begin{array}{l}\text { Change in vascularity, } \\
\text { central necrosis }\end{array}$ & $\begin{array}{l}\text { None at } 1 \\
\text { mo }\end{array}$ & None \\
\hline $\begin{array}{l}\text { Lakhtakia et al. } \\
(2016)^{8}\end{array}$ & 3 & $\begin{array}{l}\text { Functional } \\
\text { PNET }\end{array}$ & $\begin{array}{c}18 \\
(14-22)\end{array}$ & $\begin{array}{c}19 \mathrm{G}, \\
\text { Starmed }\end{array}$ & $50 \mathrm{~W}$ & 1 & $\begin{array}{l}\text { Size reduced, symptom } \\
\text { improved }\end{array}$ & $\begin{array}{c}\text { None at } 12 \\
\text { mo }\end{array}$ & None \\
\hline
\end{tabular}

EUS, endoscopic ultrasound; RFA, radiofrequency ablation; PNET, pancreatic neuroendocrine tumor. 
EUS-RFA. There were no peri-procedural complications.

Lakhtakia et al. reported 3 patients with functional PNETs (insulinoma) having symptomatic hypoglycemia who underwent EUS-guided RFA using 19 G EUSRA $^{\text {TM }}$ (STARmed). ${ }^{8}$ Rapid symptomatic and biochemical improvement was observed within 2 days after RFA in all insulinoma patients, which has sustained at 2-year follow-up. ${ }^{8}$

In general, the recent preliminary experience exhibits that EUS-guided RFA is a feasible and safe treatment option for PNETs (Table 2). The real-time visualization of the needle probe in EUS helps avoid piercing of vital intervening structures. Moreover, live imaging of RFA localized within the tumor helps reduce adverse events. ${ }^{27,30-32}$ The absence of an internal cooling mechanism in the RF probes used for percutaneous or intra-operative routes may have been responsible for the higher number of reported complications. However, the same has not been observed for transmural EUS-guided RFA in the pancreas, in the absence of an internal cooling mechanism. ${ }^{33}$ In patients undergoing EUS-guided RFA for non-PNET lesions, mild early complications have been observed, which include mild abdominal pain, mild acute pancreatitis, duodenal bleed treated endoscopically without any blood transfusion, or asymptomatic peri-pancreatic fluid collection. $^{25}$

\section{TECHNICAL ASPECTS OF EUS-GUIDED RFA}

The device is passed under EUS guidance into the selected lesion crossing the smallest length of normal pancreas and avoiding crucial intervening structures (major vessel, pancreatic duct or common bile duct). The device tip is carefully placed at the far end of the target and energy is delivered. Following a variable pause, echogenic bubbles appear around the active electrode of the device, suggesting local RFA. The magnitude of the 'ablated zone' (echogenic bubbles on EUS) may vary with the wattage used, duration of and length of the active electrode. In a large lesion, the active electrode can be relocated into a non-ablated area either along the same trajectory closer to echo-endoscope or by fanning the needle, while cautiously staying away from the GI wall. Attempts should be made to start ablation from the more technically challenging area of the lesion, since visual artifacts may obscure the EUS view after first RFA. ${ }^{8}$

Overall, recent experiences demonstrate that EUS-guided RFA is a feasible and safe treatment option for a variety of pancreatic lesions. Although the technical feasibility of EUS-guided RFA for pancreatic tumors may be acceptable, translation into clinical efficacy needs a lot more experience.
In 'functional PNETs' beneficial effects with immediate relief of symptoms and biochemical improvement are seen, which can be objectively followed. Extrapolating EUS-guided RFA in 'non-functional PNETs' is the next logical step, which can be monitored by dynamic imaging to follow sustained response. There are many grey areas in the EUS RFA for pancreatic tumors-power setting, duration of application, number of RFA sessions required according to the tumor type \& size. In addition, studies looking at objective parameters (e.g., hormone levels, pancreatic protocol CT, or contrast-enhanced EUS) which can be monitored at regular intervals, both before and after EUS-guided RFA, that document sustained improvement is needed to encourage other medical sub-specialties to incorporate this technology into their regular use in the management of PNETs.

\section{CONCLUSIONS}

EUS-guided FNI using either ethanol ablation or RFA needs to be compared in a homogenous population using standard predefined inclusion and exclusion criteria. Although the technical methodology is gradually getting standardized for both, further refinement requires close attention. The final proof of the concept would come from multicenter studies comparing surgical resection with one of these minimally invasive EUS ablative methods.

Conflicts of Interest

The author has no financial conflicts of interest.

\section{REFERENCES}

1. Yao JC, Hassan M, Phan A, et al. One hundred years after "carcinoid": epidemiology of and prognostic factors for neuroendocrine tumors in 35,825 cases in the United States. J Clin Oncol 2008;26:3063-3072.

2. Kuo EJ, Salem RR. Population-level analysis of pancreatic neuroendocrine tumors $2 \mathrm{~cm}$ or less in size. Ann Surg Oncol 2013;20:2815-2821.

3. Partelli S, Bartsch DK, Capdevila J, et al. ENETS consensus guidelines for standard of care in neuroendocrine tumours: surgery for small intestinal and pancreatic neuroendocrine tumours. Neuroendocrinology 2017;105:255-265.

4. Valle JW, Eatock M, Clueit B, Gabriel Z, Ferdinand R, Mitchell S. A systematic review of non-surgical treatments for pancreatic neuroendocrine tumours. Cancer Treat Rev 2014;40:376-389.

5. Levy MJ, Thompson GB, Topazian MD, Callstrom MR, Grant CS, Vella A. US-guided ethanol ablation of insulinomas: a new treatment option. Gastrointest Endosc 2012;75:200-206.

6. Park DH, Choi JH, Oh D, et al. Endoscopic ultrasonography-guided ethanol ablation for small pancreatic neuroendocrine tumors: results of a pilot study. Clin Endosc 2015;48:158-164.

7. Qin SY, Lu XP, Jiang HX. EUS-guided ethanol ablation of insulinomas: case series and literature review. Medicine (Baltimore) 2014;93:e85.

8. Lakhtakia S, Ramchandani M, Galasso D, et al. EUS-guided radiofrequency ablation for management of pancreatic insulinoma by using a novel 
needle electrode (with videos). Gastrointest Endosc 2016;83:234-239.

9. Song TJ, Seo DW, Lakhtakia S, et al. Initial experience of EUS-guided radiofrequency ablation of unresectable pancreatic cancer. Gastrointest Endosc 2016;83:440-443.

10. Pai M, Habib N, Senturk H, et al. Endoscopic ultrasound guided radiofrequency ablation, for pancreatic cystic neoplasms and neuroendocrine tumors. World J Gastrointest Surg 2015;7:52-59.

11. Shiina S, Teratani T, Obi S, Hamamura K, Koike Y, Omata M. Percutaneous ethanol injection therapy for liver tumors. Eur J Ultrasound 2001;13:95-106.

12. Barclay RL, Perez-Miranda M, Giovannini M. EUS-guided treatment of a solid hepatic metastasis. Gastrointest Endosc 2002;55:266-270.

13. Günter E, Lingenfelser T, Eitelbach F, Müller H, Ell C. EUS-guided ethanol injection for treatment of a GI stromal tumor. Gastrointest Endosc 2003;57:113-115.

14. Artifon EL, Lucon AM, Sakai P, et al. EUS-guided alcohol ablation of left adrenal metastasis from non-small-cell lung carcinoma. Gastrointest Endosc 2007;66:1201-1205

15. Aslanian H, Salem RR, Marginean C, Robert M, Lee JH, Topazian M. EUS-guided ethanol injection of normal porcine pancreas: a pilot study. Gastrointest Endosc 2005;62:723-727.

16. Matthes K, Mino-Kenudson M, Sahani DV, Holalkere N, Brugge WR. Concentration-dependent ablation of pancreatic tissue by EUS-guided ethanol injection. Gastrointest Endosc 2007;65:272-277.

17. Jürgensen C, Schuppan D, Neser F, Ernstberger J, Junghans U, Stölzel U. EUS-guided alcohol ablation of an insulinoma. Gastrointest Endosc 2006;63:1059-1062.

18. Muscatiello N, Salcuni A, Macarini L, et al. Treatment of a pancreatic endocrine tumor by ethanol injection guided by endoscopic ultrasound. Endoscopy 2008;40 Suppl 2:E258-E259.

19. Deprez PH, Claessens A, Borbath I, Gigot JF, Maiter D. Successful endoscopic ultrasound-guided ethanol ablation of a sporadic insulinoma. Acta Gastroenterol Belg 2008;71:333-337.

20. Vleggaar FP, Bij de Vaate EA, Valk GD, Leguit RJ, Siersema PD. Endoscopic ultrasound-guided ethanol ablation of a symptomatic sporadic insulinoma. Endoscopy 2011;43 Suppl 2 UCTN:E328-E329.

21. Goldberg SN, Mallery S, Gazelle GS, Brugge WR. EUS-guided radiofre- quency ablation in the pancreas: results in a porcine model. Gastrointest Endosc 1999;50:392-401.

22. Carrara S, Arcidiacono PG, Albarello L, et al. Endoscopic ultrasound-guided application of a new hybrid cryotherm probe in porcine pancreas: a preliminary study. Endoscopy 2008;40:321-326.

23. Kim HJ, Seo DW, Hassanuddin A, et al. EUS-guided radiofrequency ablation of the porcine pancreas. Gastrointest Endosc 2012;76:1039-1043.

24. Lakhtakia S, Seo DW. Endoscopic ultrasonography-guided tumor ablation. Dig Endosc 2017;29:486-494.

25. Alvarez-Sánchez MV, Napoléon B. Review of endoscopic radiofrequency in biliopancreatic tumours with emphasis on clinical benefits, controversies and safety. World J Gastroenterol 2016;22:8257-8270.

26. Armellini E, Crinò SF, Ballarè $M$, Occhipinti P. Endoscopic ultrasound-guided radiofrequency ablation of a pancreatic neuroendocrine tumor. Endoscopy 2015;47 Suppl 1 UCTN:E600-E601.

27. Arcidiacono PG, Carrara S, Reni M, et al. Feasibility and safety of EUS-guided cryothermal ablation in patients with locally advanced pancreatic cancer. Gastrointest Endosc 2012;76:1142-1151.

28. Rossi S, Viera FT, Ghittoni G, et al. Radiofrequency ablation of pancreatic neuroendocrine tumors: a pilot study of feasibility, efficacy, and safety. Pancreas 2014;43:938-945.

29. Weigt J, Kandulski A, Malfertheiner P. Endoscopic intraductal radiofrequency ablation of remnant intrapapillary mucinous neoplasm with acute hemorrhage after incomplete surgical resection. Endoscopy 2014;46 Suppl 1 UCTN:E489-E490.

30. Matsui Y, Nakagawa A, Kamiyama Y, Yamamoto K, Kubo N, Nakase Y. Selective thermocoagulation of unresectable pancreatic cancers by using radiofrequency capacitive heating. Pancreas 2000;20:14-20.

31. Wu Y, Tang Z, Fang H, et al. High operative risk of cool-tip radiofrequency ablation for unresectable pancreatic head cancer. J Surg Oncol 2006;94:392-395.

32. Elias D, Baton O, Sideris L, Lasser P, Pocard M. Necrotizing pancreatitis after radiofrequency destruction of pancreatic tumours. Eur J Surg Oncol 2004;30:85-87.

33. Girelli R, Frigerio I, Salvia R, Barbi E, Tinazzi Martini P, Bassi C. Feasibility and safety of radiofrequency ablation for locally advanced pancreatic cancer. Br J Surg 2010;97:220-225. 